\title{
Use of vonoprazan for management of systemic sclerosis-related gastroesophageal reflux disease
}

\author{
MAIKO TABUCHI $^{1}$, HITOMI MINAMI ${ }^{1}$, YUKO AKAZAWA $^{1,2}$, MIWA ASHIDA $^{3}$, \\ TOSHIHIDE HARA ${ }^{4}$, KUNIHIRO ICHINOSE ${ }^{5}$, MOTO KITAYAMA ${ }^{1}$, KEIICHI HASHIGUCHI $^{1}$, \\ KAYOKO MATSUSHIMA ${ }^{1}$, NAOYUKI YAMAGUCHI ${ }^{1}$, FUMINAO TAKESHIMA ${ }^{1}$, \\ HISAYOSHI KONDO ${ }^{6}$, ATSUSHI KAWAKAMI ${ }^{5}$ and KAZUHIKO NAKAO ${ }^{1}$
}

Departments of ${ }^{1}$ Gastroenterology and Hepatology, ${ }^{2}$ Pathology and ${ }^{3}$ Dermatology, Nagasaki University Graduate School of Biomedical Science, Nagasaki 852-8501;

${ }^{4}$ Department of Dermatology, Japan Community Healthcare Organization, Isahaya General Hospital, Isahaya, Nagasaki 854-8501; ${ }^{5}$ Department of Immunology and Rheumatology, Nagasaki University Graduate School of Biomedical Science, Nagasaki 852-8501; ${ }^{6}$ Division of Scientific Data Registry, Atomic Bomb Disease and Hibakusya Medicine Unit, Atomic Bomb Disease Institute, Nagasaki University, Nagasaki 852-8523, Japan

Received June 12, 2020; Accepted November 13, 2020

DOI: $10.3892 / b r .2020 .1401$

\begin{abstract}
Gastroesophageal reflux disease (GERD) in systemic sclerosis (SSc) can significantly reduce a patient's quality of life. GERD in SSc is occasionally resistant to conventional anti-acid treatment. Vonoprazan is an $\mathrm{H}^{+} / \mathrm{K}^{+}$-ATPase blocker that is approved in Japan for treatment of GERD. The aim of the present study was to evaluate the efficacy of vonoprazan in SSc-related GERD. The frequency scale for symptoms of GERD (FSSG) scores were collected before and after vonoprazan treatment in $15 \mathrm{SSc}$ patients with GERD. Additionally, endoscopic esophagogastroduodenoscopy was performed in select patients. Conventional proton pump inhibitors or histamine-2 receptor antagonists had been previously administered in $93 \%$ (14/15) of the patients. Although the baseline esophagogastroduodenoscopy examination did not show severe erosion in the majority of patients, the mean total FSSG score before vonoprazan treatment was notably high $(25.2 \pm 10.7)$ compared to a normal score of $<8$. After vonoprazan treatment, the FSSG score decreased to 9.6 \pm 7.0 . The mean improvement rate of the total FSSG, acid reflux and dysmotility scores were $60.8 \pm 21.2 \%(\mathrm{P}=0.0004), 67.3 \pm 24.8 \%(\mathrm{P}<0.0001)$ and $55.4 \pm 26.0 \%(\mathrm{P}=0.0022)$, respectively. These results suggest that vonoprazan may be a potentially effective treatment for GERD in patients with SSc.
\end{abstract}

Correspondence to: Dr Maiko Tabuchi, Department of Gastroenterology and Hepatology, Nagasaki University Graduate School of Biomedical Science, 1-7-1 Sakamoto, Nagasaki 852-8501, Japan E-mail:m.tabuchi@nagasaki-u.ac.jp

Key words: vonoprazan, gastroesophageal reflux disease, systemic sclerosis, frequency scale for symptoms of gastroesophageal reflux disease, proton pump inhibitor

\section{Introduction}

Systemic sclerosis $(\mathrm{SSc})$ is an autoimmune disease resulting in tissue fibrosis, vascular angiopathy and visceral damage (1). Microvascular changes in SSc can lead to muscle fibrosis in the digestive system (2), which results in esophageal dysmotility in $65-95 \%$ of patients (3-9). SSc patients often present with lower esophageal sphincter (LES) dysfunction and low or absent primary and secondary peristalsis in the distal esophagus (2), leading to gastroesophageal acid reflux and reduced gastric acid clearance from the esophagus. As a result, gastroesophageal reflux disease (GERD), a condition characterized by erosions seen on endoscopy, and symptoms such as heartburn, occur in 45-80\% of patients with SSc (4,9-12). Furthermore, the presence of esophageal symptoms may predict esophageal dysmotility in SSc patients (7).

GERD management includes changes to lifestyle and administration of proton pump inhibitors (PPIs), H2 receptor antagonists (H2RA), or prokinetic agents to improve gastric emptying (13). The last option is surgical intervention (such as fundoplication or Roux-en-Y reconstruction method) (13). PPIs are currently the first-line therapy for patients with GERD, including SSc patients. Whilst conventional PPIs have been reported to generally work well in GERD patients with SSc (14), another study indicated that patients developed esophageal symptoms, such as heartburn (77.4\%), dysphagia (14.3\%), and nausea and vomiting $(9.8 \%)$ after prolonged treatment with the conventional PPI, omeprazole (20 or $40 \mathrm{mg} /$ day) (15). Thus, it is crucial to develop treatments that can effectively treat GERD in all patients with SSc.

Vonoprazan is a first-in-class PPI that was approved for use by the Ministry of Health, Labour and Welfare of Japan in February 2015 (16). Vonoprazan binds to the $\mathrm{H}^{+} / \mathrm{K}^{+}$-ATPase of parietal cells in a $\mathrm{K}^{+}$-competitive manner, and unlike conventional PPIs, functions in the absence of an acidic environment. Furthermore, vonoprazan accumulates in parietal cells and 
provides more consistent acid suppression compared with other PPIs (16). Previous studies have demonstrated the efficacy of vonoprazan for the treatment of refractory GERD in patients without SSc $(17,18)$; however, the use of vonoprazan in patients with SSc complicated by GERD has not been investigated, to the best of our knowledge. Thus, the aim of the present study was to evaluate the efficacy of vonoprazan for the treatment of GERD in patients with SSc.

\section{Materials and methods}

Patients. The Nagasaki University Hospital Ethics Committee approved the present study protocol (approval nos. 16062704 and 17082135). All investigations were performed in accordance with the 1964 Declaration of Helsinki (19). The information was obtained only from anonymized electronic medical records. According to the guidelines of the Nagasaki University Hospital Ethical Committee's official disclosure system, if the subject of the study has minimal risk, the patient may choose to opt out of a study through our institution's homepage (mh. nagasaki-u.ac.jp/research/rinsho/patients/open.html). Informed consent was obtained from all patients in this study.

The present study was performed between July 1, 2016 and May 31, 2017 at Nagasaki University Hospital. A total of 17 outpatients with GERD who received vonoprazan treatment at the Department of Dermatology, Immunology and Rheumatology were retrospectively recruited. The exclusion criteria included age (patients $<20$ and $>90$ years of age), and those who did not provide informed consent. Of the 17 enrolled patients with GERD, two patients who were eventually diagnosed with morphea or mixed connective tissue disease, during the course of treatment, were excluded. Thus, 15 participants were recruited in the final cohort $[1$ male $(6 \%)$ and 14 females (94\%); median age, 64 years (range: 36-78); limited cutaneous (lc)SSc, $n=11$ (73\%); diffuse cutaneous (dc)SSc, $n=4(26 \%)]$. GERD treatment scores with a frequency scale for symptoms of GERD (FSSG) (20), and on and off (previous) vonoprazan treatment was recorded. Characteristics of participating patients and the endoscopic findings of GERD were collected from electronic records. The mean observation period of endoscopy was 7.5 weeks (range, 0-32 weeks) before vonoprazan treatment. History of the use of various anti-acids were found, but only previous use the anti-acids of vonoprazan was recorded. Conventional PPIs and H2RA included esomeprazole $20 \mathrm{mg} /$ day, lansoprazole $30 \mathrm{mg} /$ day, rabeprazole $10 \mathrm{mg} /$ day and famotidine $20 \mathrm{mg} /$ day. Duration of treatment with previous PPIs was 20 to 436 weeks and with H2RA treatment it was 8 weeks.

In 2 patients out of 15, in addition to recording the above information, 24-h pH monitoring and high-resolution manometry (HRM) was performed, both before and after 12 weeks of vonoprazan therapy. Patients were observed for 4-28 weeks after beginning vonoprazan. Patients who underwent the 24-h $\mathrm{pH}$ monitoring and HRM provided informed consent before participating in the present study.

Diagnosis of SSc. Patients were categorized into dcSSc and lcSSc groups, according to the range of skin hardening and other factors, including the presence of auto-antibodies and complications (21). Anti-topoisomerase-I antibody and anti-RNA polymerase antibody positivity suggested
dcSSc, and anticentromere antibody positivity confirmed lcSSc. Additionally, the period between diagnosis and occurrence of SSc, the modified Rodnan total skin thickness score (MRSS), Raynaud's phenomenon, digital ulcer, pulmonary arterial hypertension (diagnosed by cardiac echocardiography) and interstitial lung disease (diagnosed by high-resolution computed tomography) was recorded.

Treatment with vonoprazan. The dose of vonoprazan administered to patients (either 10 or $20 \mathrm{mg} /$ day) was determined by the patient's attending physician. A total of 7 patients were treated with $20 \mathrm{mg}$ vonoprazan from the beginning of the study and 8 patients were started on $10 \mathrm{mg} /$ day vonoprazan, of which, 4 patients were increased to $20 \mathrm{mg} /$ day after $4-17$ weeks, due to insufficient effects at $10 \mathrm{mg} /$ day.

FSSG. The FSSG score developed by Kusano et al (20), has been reported to correctly identify GERD and reflect disease severity and treatment efficacy in Japanese GERD patients (22). Patients filled in the questionnaire before vonoprazan treatment (baseline) in all patients. A follow-up questionnaire was filled in after at least 4 weeks of treatment (range, 4-28 weeks follow-up). The total FSSG score was 48 points, and scores $>8$ indicated GERD.

Esophageal HRM and 24-h pH monitoring. A pH electrode catheter (Catheter, SA100; Pocket Monitor, GMMS-200pH; Star Medical) sensor was placed $5 \mathrm{~cm}$ above the esophagogastric junction under X-ray guidance and maintained for $24 \mathrm{~h}$. The data was analyzed using pH.dll software (Star Medical). The Demeester score (23), acid exposure time (AET; defined as the ratio of the total time the distal esophageal $\mathrm{pH}$ was $<4$ to the total monitoring time), number of reflux events lasting $>5 \mathrm{~min}$ and the total number of acid reflux events were recorded. Esophageal motility was assessed using HRM (Catheter, PD1236K, Pocket Monitor: GMMS-4000, Star Medical) according to the Chicago Classification system version 3.0 (24).

Statistical analysis. Data was analyzed with JMP software (ver. 15.0.0; SAS institute). Patient's data are presented as the median (range). The FSSG scores before and after vonoprazan treatment were compared using a Wilcoxon's paired rank sum test. For comparison of the improvement rate between the starting dose of 10 and $20 \mathrm{mg}$ of vonoprazan and for the comparison of digestive involvement with SSc patients and the improvement rate of vonoprazan treatment, the Wilcoxon's unpaired rank sum test was used. A Kruskal-Wallis test followed by a post-hoc Dunn's test was used for comparison between the improvement rate of vonoprazan and each SSc grade. $\mathrm{P}<0.05$ was considered to indicate a statistically significant difference. The improvement in the FSSG scores was adjusted to account for differences in the duration of disease according to the following equation: Improvement rate $=$ (before vonoprazan treatment score-post-treatment score)/before vonoprazan treatment score x100.

\section{Results}

Patient background. A total of 15 outpatients with SSc complicated by GERD were treated with vonoprazan during 
Table I. Characteristics of SSc patients with gastroesophageal reflux disease.

\begin{tabular}{lc}
\hline Characteristic & Value, $\mathrm{n}=15$ \\
\hline Age, median (range) & $64(36-78)$ \\
Sex, $\mathrm{n}(\%)$ & \\
Male & $1(6)$ \\
Female & $14(93)$ \\
SSc duration, median years (range) & $10(0-19)$ \\
SSc subtype & \\
dcSSc, $\mathrm{n}(\%)$ & $4(26)$ \\
lcSSc, $\mathrm{n}(\%)$ & $11(73)$ \\
MRSS, median score (range) & $5(0-26)$ \\
Autoantibody subtype & \\
Topoisomerase-1, $\mathrm{n}(\%)$ & $3(20)$ \\
Centromere, $\mathrm{n}(\%)$ & $4(26)$ \\
RNA polymerase, $(\%)$ & $2(13)$ \\
Other, unknown, $\mathrm{n}(\%)$ & $6(40)$ \\
Pulmonary hypertension, $\mathrm{n}(\%)$ & $2(13)$ \\
Interstitial lung disease, $\mathrm{n}(\%)$ & $7(46)$ \\
Raynaud's syndrome, $\mathrm{n}(\%)$ & $13(86)$ \\
Digital ulcer, $\mathrm{n}(\%)$ & $3(20)$ \\
Symptoms, $\mathrm{n}(\%)$ & \\
Heartburn & $14(93)$ \\
Dysphagia & $6(40)$ \\
Epigastric pain & $6(40)$ \\
Vomiting & $5(33)$ \\
Constipation & $4(26)$ \\
Weight loss & $1(6)$ \\
Previous therapeutic drug, $\mathrm{n}(\%)$ & \\
Histamine-2 receptor antagonist & $1(7)$ \\
Proton pump inhibitor & $13(86)$ \\
\hline
\end{tabular}

SSc, systemic sclerosis; dcSSc, diffuse cutaneous systemic sclerosis; lcSSc, limited cutaneous systemic sclerosis; MRSS, Modified Rodnan skin score.

the study period. A total of 2 patients (13\%) had pulmonary hypertension and 7 (46\%) had pulmonary fibrosis. The median (range) MRSS was 7.7 (0-26). Symptoms of Raynaud's phenomenon occurred in $13(86 \%)$ patients and $3(20 \%)$ patients had a digital ulcer (Table I). Gastrointestinal symptoms included heartburn, epigastric pain, dysphagia, vomiting, constipation and weight loss in 14 (93\%), 6 (40\%), 6 (40\%), 5 (33\%), 4 (26\%) and $1(6 \%)$ patient, respectively. The mean duration of SSc was $10.2 \pm 5.3$ years. In the patients, 8 started vonoprazan at $10 \mathrm{mg} /$ day, whereas 7 started at $20 \mathrm{mg} /$ day. Of the 15 patients, 14 patients (93\%) had previously been treated with conventional PPIs and H2RA without relief (Fig. S1A). Only, 1 patient had discontinued the PPI despite recurring GERD symptoms, due to a fear of the side effects. The median period of conventional PPIs and H2RA treatment was 122 weeks (range, 8-436 weeks).

Amongst all the 15 patients, previous medications included esomeprazole $(n=9,60 \%)$, lansoprazole $(n=2,13 \%)$, rabeprazole
Table II. Gastroesophageal reflux disease grade according to the modified LA classification before vonoprazan treatment.

\begin{tabular}{lc}
\hline Modified LA grade before treatment & $\mathrm{n},(\%)^{\mathrm{a}}$ \\
\hline $\mathrm{N} / \mathrm{M}$ & $8(72)$ \\
$\mathrm{A} / \mathrm{B}$ & $3(27)$ \\
$\mathrm{C} / \mathrm{D}$ & $0(0)$ \\
\hline
\end{tabular}

${ }^{\mathrm{a}}$ Of the 15 patients, 11 underwent an endoscopy before vonozapran treatment (0-32 weeks before vonoprazan treatment). N, no changes at the EGJ; M, minimal change without mucosal break at the EGJ; $\mathrm{A},<5 \mathrm{~mm}$ erosion at the $\mathrm{EGJ} ; \mathrm{B},>5 \mathrm{~mm}$ erosion at the EGJ or in the lower esophagus and no connection to the next erosion; C, >2 lines of erosion at the EGJ with connection to another erosion, D, circumferential involvement or stricture at the EGJ. LA, Los Angeles; EGJ, esophagogastric junction.

$(\mathrm{n}=2,13 \%)$ and famotidine $(\mathrm{n}=1,7 \%)$, with one patient $(7 \%)$ not having received any prior treatment (Fig. S1A). Approximately $75 \%$ of patients had previously received a prokinetic agent (mosapride citrate or metoclopramide) (Fig. S1B).

A total of 11 patients underwent endoscopy and the Los Angeles (LA) grading (25) of GERD before initiation of vonoprazan treatment. A majority of patients $(n=8,72 \%)$ had a normal or slightly inflamed esophageal mucosa (LA Grade N/M) regardless of symptom severity (Table II). A total of 3 patients (27\%) had slight to moderate esophagitis (LA grade $\mathrm{A} / \mathrm{B}$ ), and none had severe esophagitis (LA grade C).

FSSG score assessment before and after vonoprazan. The mean baseline total FSSG, acid reflux and dysmotility scores (standard deviation) were 25.2 (10.7), 14.1 (6.6) and 11.1 (4.6), respectively, before vonoprazan treatment (Table III). The mean of all three scores significantly improved after 4-28 weeks of vonoprazan treatment in patients receiving either 10 or $20 \mathrm{mg}$ /day (mean scores, 9.6, 4.6 and 4.8, respectively; Table III). A significant improvement in the mean total and acid reflux FSSG score was observed: $60.8 \pm 21.2$ and $67.3 \pm 24.8 \%$, respectively (Table III). Somewhat unexpectedly, the mean dysmotility score also showed an improvement (55.4 $\pm 26.0 \%)$.

In the 7 patients who received vonoprazan $20 \mathrm{mg} /$ day from the beginning, the mean FSSG total, acid reflux and dysmotility score improvement rates were $65.7,73.5$ and $60.4 \%$, respectively. In contrast, in the 8 patients who started treatment at $10 \mathrm{mg} /$ day, the median improvement rates were modest at $29.9,39.1$ and $25.8 \%$, respectively, which was significantly less compared with those who began treatment with $20 \mathrm{mg} /$ day (Fig. 1).

Of the 8 patients who started at $10 \mathrm{mg}$, in 4 patients, this dose was ineffective and were thus increased to $20 \mathrm{mg}$ vonoprazan, which was effective. The remaining 4 patients remained on $10 \mathrm{mg}$ owing to a sufficient beneficial effect at that dose.

Regarding whether symptoms of digestive tract involvement in SSc other than reflux esophagitis (dysphagia, epigastric pain, vomiting, weight loss and constipation) affected the effects of vonoprazan on GERD symptoms in patients with dysphagia, GERD symptoms showed less improvement, whereas the presence of other symptoms did not affect the 
Table III. FSSG scores in patients with systemic sclerosis-related GERD before and after vonoprazan treatment.

\begin{tabular}{|c|c|c|c|c|}
\hline FSSG score & Before vonoprazan & After vonoprazan ${ }^{c}$ & Mean improvement rate ${ }^{\mathrm{d}} \pm \mathrm{SD}$ & $\mathrm{P}$-value \\
\hline Total score, mean (SD) & $25.2(10.7)$ & $9.6(7.0)$ & $60.8 \% \pm 21.2$ & $0.0004^{\mathrm{b}}$ \\
\hline Acid reflux score, mean (SD) & $14.1(6.6)$ & $4.6(5.1)$ & $67.3 \% \pm 24.8$ & $<0.0001^{\mathrm{b}}$ \\
\hline Dysmotility score, mean (SD) & $11.1(4.6)$ & $4.8(3.5)$ & $55.4 \% \pm 26.0$ & $0.0022^{\mathrm{a}}$ \\
\hline
\end{tabular}

${ }^{\mathrm{a}} \mathrm{P}<0.05,{ }^{\mathrm{b}} \mathrm{P}<0.001 .{ }^{\mathrm{c}}$ Vonoprazan dose, 10 or $20 \mathrm{mg} /$ day. ${ }^{\mathrm{d}}$ Improvement rate=(before vonoprazan score-after vonoprazan treatment score)/before vonoprazan score x100 (\%). The duration of observation was 4-28 weeks after vonoprazan treatment. ${ }^{\mathrm{e}} \mathrm{A}$ Wilcoxon paired test was used. GERD, gastroesophageal reflux disease; FSSG, frequency score for symptoms of GERD; SD, standard deviation.
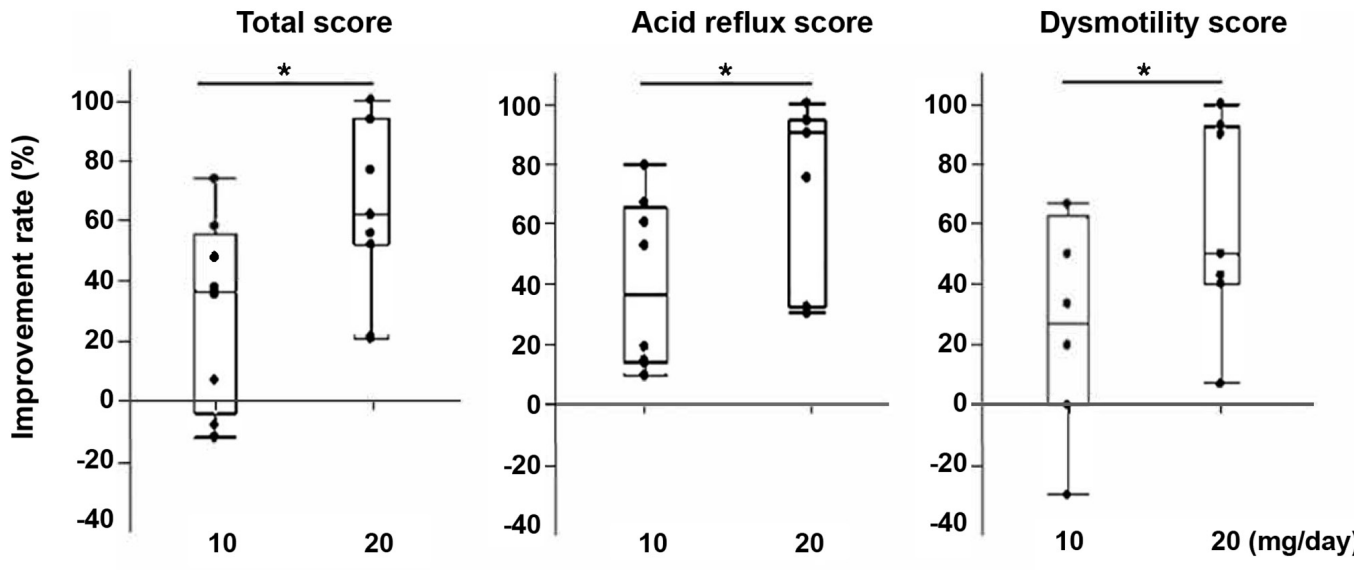

Starting dose of vonoprazan

Figure 1. Comparison of improvement rates between a starting dose of 10 or $20 \mathrm{mg}$ vonoprazan in all 15 patients. The scatter diagram compares the improvement rates for the difference in initial vonoprazan dose; $10 \mathrm{mg}$ (4-17 weeks after vonoprazan) vs. $20 \mathrm{mg}$ (6-28 weeks after vonoprazan). The symptom improvement rates of $20 \mathrm{mg}$ were significantly higher than those of $10 \mathrm{mg}$ in all three categories. Thus, a top-down therapy of $20 \mathrm{mg}$ vonoprazan may be better than starting at $10 \mathrm{mg}$. ${ }^{*} \mathrm{P}<0.05$.

effect of vonoprazan (Fig. 2). Patients with dysphagia had a significantly lower rate of improvement.

Side effects (diarrhea, nausea, vomiting, skin rash and erythema multiforme) of vonoprazan were not reported in the follow-up period.

Additionally, association analysis between the grade of SSc (26) and the improvement rate of vonoprazan (using a Kruskal-Wallis test) was assessed. However, there were no statistically significant differences amongst these (Fig. 3).

Assessment of 24-h pH monitoring and HRM in two patients. In two cases, 24-h pH monitoring and HRM were recorded at baseline and after 12 weeks of vonoprazan treatment. The baseline to after treatment total FSSG scores decreased from 30 to 7 (Case 1) and 9 to 4 (Case 2), and endoscopic examination revealed evidence of mild erosions in both cases. After $20 \mathrm{mg} /$ day vonoprazan treatment, both patients showed improved endoscopic findings (Fig. 4A). These two patients had severe reflux observed with $24-\mathrm{h} \mathrm{pH}$ monitoring, indicating excessive AET (76 and 97\%). Notably, supine position AET was increased (77 and 87\%) and the baseline Demeester scores were also high (Fig. 4B). The AET and Demeester scores showed a trend towards mild improvement following treatment (Fig. 4B).

HRM revealed absent contractility and hypotensive LES (3.6 and $3.8 \mathrm{mmHg}$, respectively; Fig. 4C). No significant changes in esophageal pressure topography of HRM was observed between before and after treatment with vonoprazan, despite the improved FSSG motility disorder scores in each patient (12 to 6 and 5 to 3 , respectively).

\section{Discussion}

To the best of our knowledge, the present study is the first to describe the effects of vonoprazan in a SSc patient population. The majority of SSc patients in the present study had experienced severe gastroesophageal symptoms that persisted regardless of treatment with conventional PPIs (Table I). Although all patients had symptoms of GERD, the endoscopic evidence of esophagitis tended to be modest even in those with severe symptoms, which is in contrast to a report by Thonhofer et al (27), who showed that $77 \%$ of asymptomatic SSc patients had esophageal erosions. This difference in findings may be due to the administration of PPI prior to initiation of vonoprazan treatment in the majority of the patients in the current study, and suggest that physicians should not judge the severity of esophageal manifestations based on mucosal erosion alone.

The primary scoring systems for GERD is the FSSG as well as the modified FSSG (28). FSSG primarily takes into consideration reflux symptoms, whereas the modified FSSG score is beneficial for the differentiation of functional dyspepsia and 

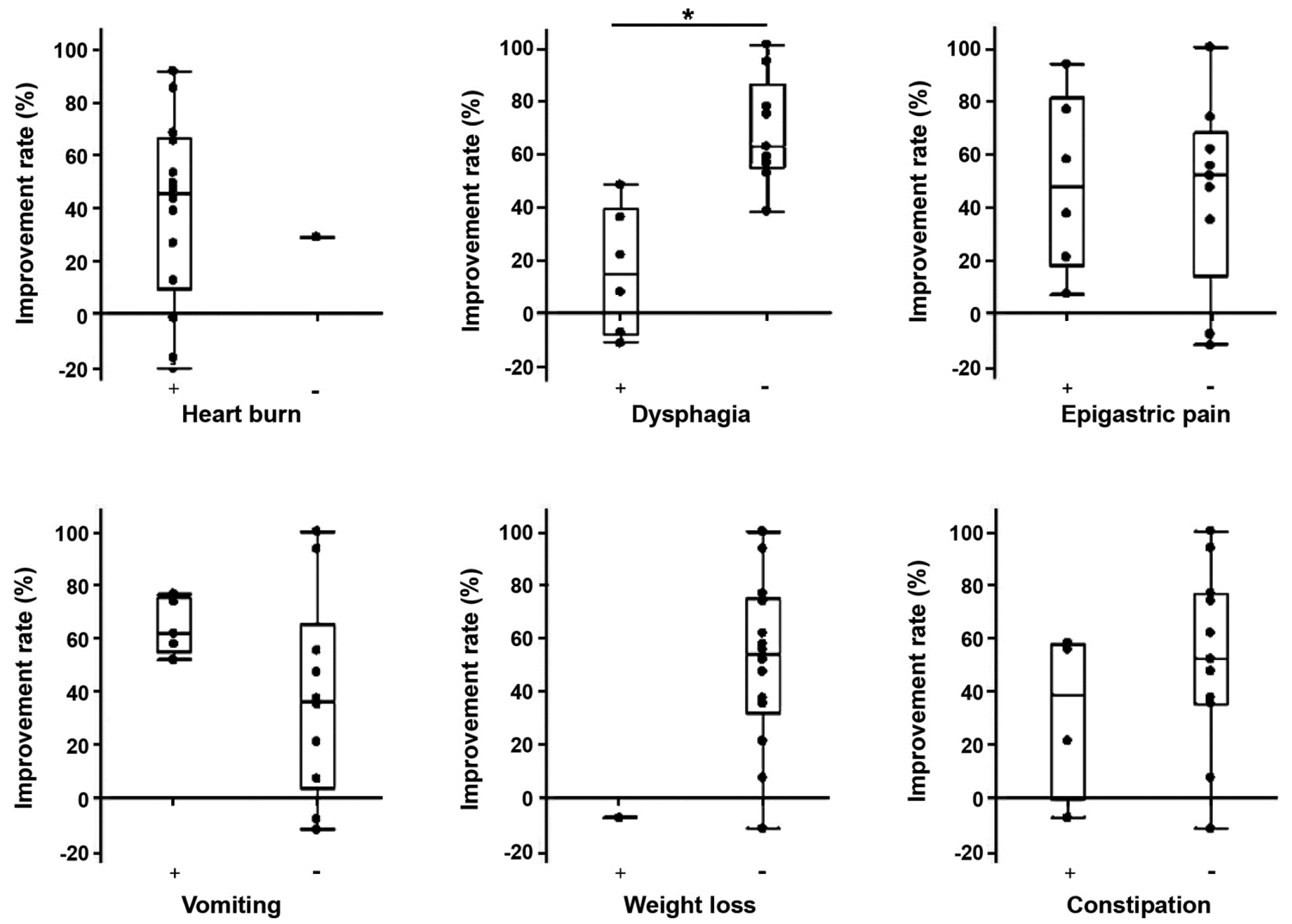

Figure 2. A comparison of digestive involvement with SSc patients and the improvement rate of FSSG with vonoprazan treatment. The scatter diagram shows the effect of vonoprazan on GERD symptoms (the improvement rate of vonoprazan) under the presence or absence of general gastrointestinal symptoms in SSc. In the presence of dysphagia, the improvement rate was significantly lower. The duration of the observation period was 4-28 weeks after vonoprazan treatment. ${ }^{*} \mathrm{P}<0.05$. SSc, systemic sclerosis; GERD, gastroesophageal reflux disease; FSSG, frequency scale for symptoms of GERD.
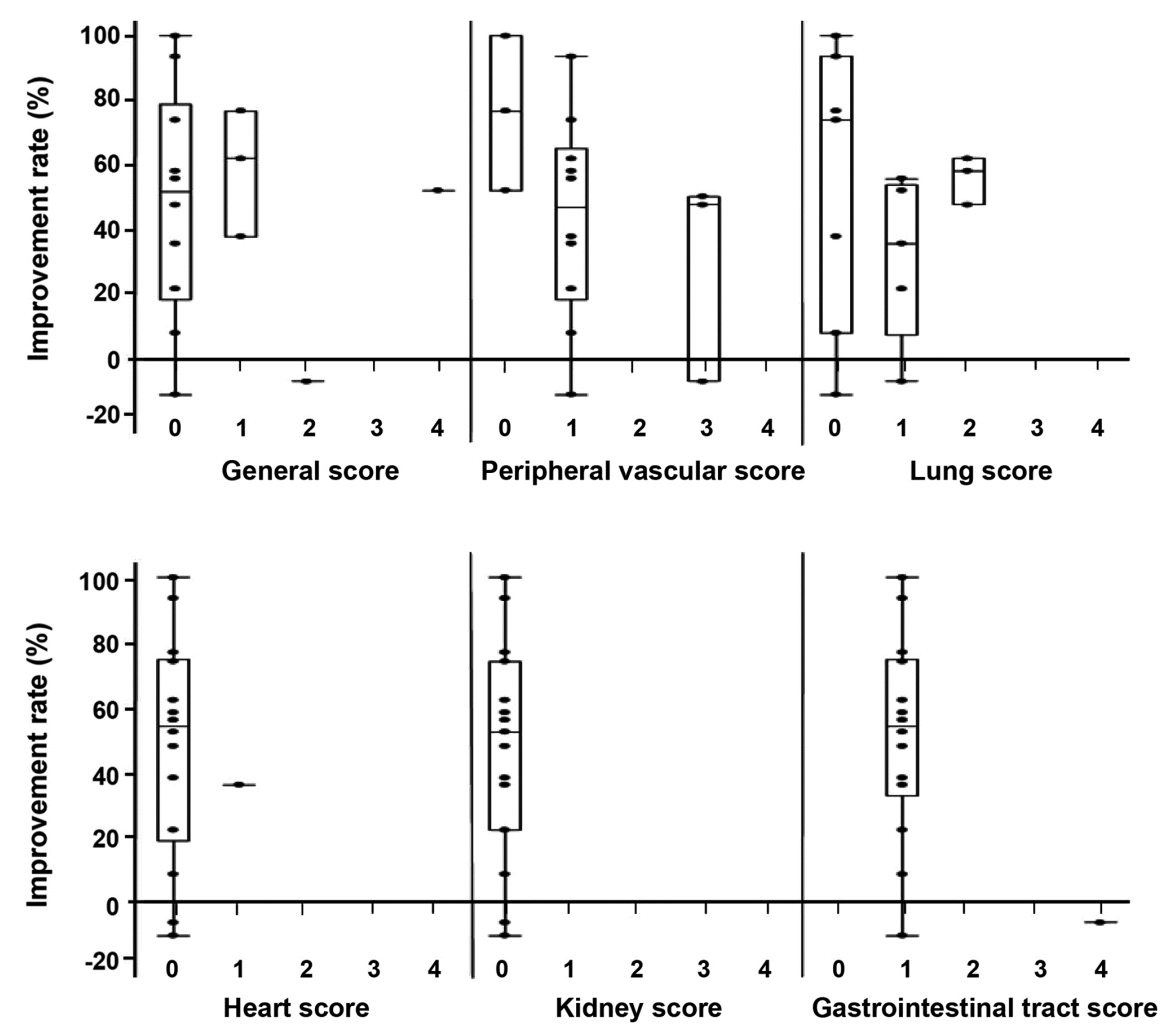

Figure 3. Association analysis between SSc grade and the improvement rate of FSSG following treatment with vonoprazan. The scatter diagram shows a comparison of the severity of each organ disorder in SSc patients and improvement rate following treatment with vonoprazan. The period of observation was 4-28 weeks after vonoprazan treatment. There were no significant differences found in any of the comparisons. SSc, systemic sclerosis; FSSG, frequency scale for symptoms of gastroesophageal reflux disease. 


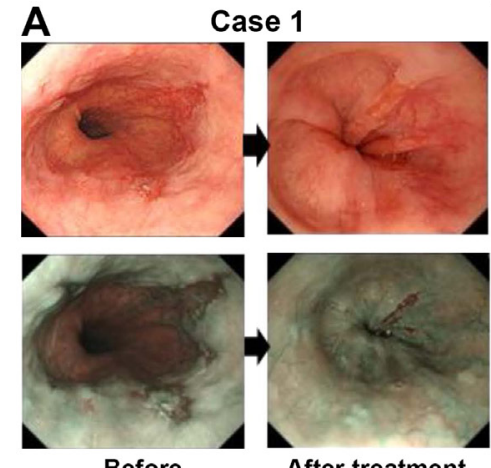

Before
After treatment

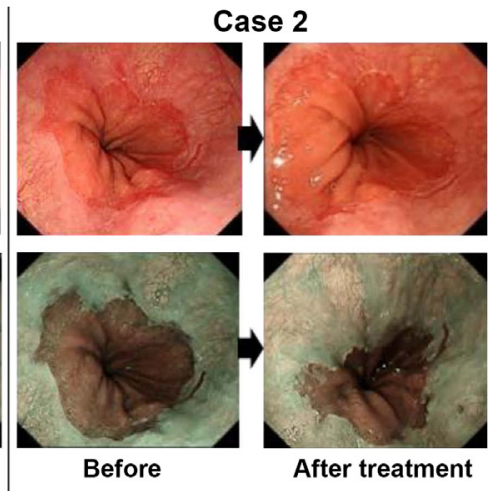

B
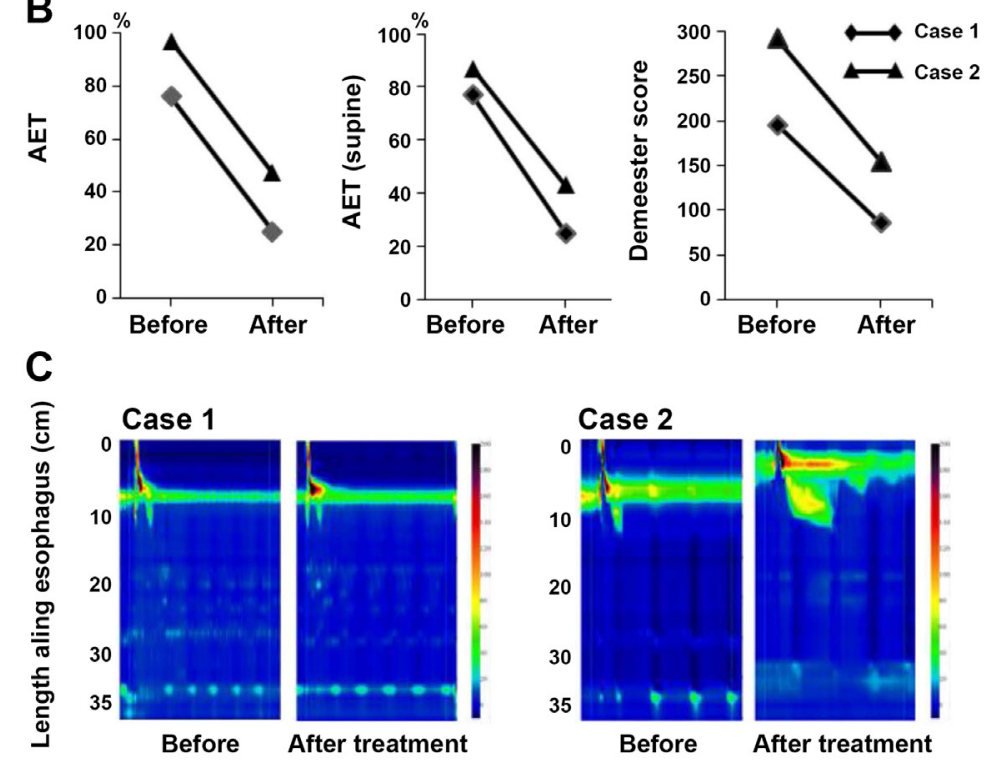

Figure 4. Results of endoscopic findings, 24-h pH monitoring and HRM. (A) Endoscopic changes before and after vonoprazan treatment using white light and narrow band imaging. (B) Comparison of individual 24- $\mathrm{h} \mathrm{pH}$ monitoring results at baseline and after vonoprazan treatment. $\mathrm{n}=2$. (C) HRM imaging with pressure topography plots at baseline and after treatment. $n=2$. The images were selected at representative points from each examination. HRM, high-resolution manometry; AET, acid exposure time.

GERD (28). In the present study, FSSG was used as the patient was determined to have GERD and the aim of the study was to assess the improvement of the reflux symptoms.

In the present study, endoscopic mucosal lesions were mild despite the severity of GERD symptoms. Conventional PPIs had already been administrated in the majority of patients, which may have resulted in mild or no mucosal damage of the esophago-gastric junction, despite the presence of GERD symptoms (29). Although grade C/D patients were not included in the present study, vonoprazan has been reported to have better effects with improved erosion in severe patients compared to PPI (30).

The effect of conventional PPIs is influenced by three CYP2C19 genotype classes: Extensive metabolizer (EM), intermediate metabolizer (IM) and poor metabolizer (31). The majority of Japanese individuals are EMs and IMs; thus, a sufficient antacid effect cannot be obtained with conventional PPIs (32). Vonoprazan does not exhibit this CYP2C19 genotype dependency as it is primarily metabolized by CYP3A4. Therefore, vonoprazan has been shown to exhibit a more constant and rapid acid suppressive effect than conventional PPIs in Japanese patients $(16,32)$. As previous studies on vonoprazan were performed primarily in Japan, further research in patients of other nationalities is required.
A previous study showed that the 24-h intra-gastric $\mathrm{pH}>4$ holding time ratio (HTR) of vonoprazan is extremely high (33); in healthy Japanese men receiving treatment with 20 or $40 \mathrm{mg}$ /day vonoprazan for 7 days, the intra-gastric $\mathrm{pH}>4$ HTRs were 83.4 and $100 \%$, respectively. Notably, the night-time intra-gastric $\mathrm{pH}>4$ HTR with $40 \mathrm{mg} /$ day vonoprazan was $100 \%$ in Japanese patients (34). The present study showed that vonoprazan markedly reduced GERD symptoms as demonstrated by improved FSSG scores, particularly the acid reflux score. In fact, 24-h pH monitoring revealed a trend toward decreased AET when lying down in 2 patients. In SSc patients, gravity serves a major role in the esophageal acid clearance time, and delayed acid clearance in the supine position may suggest initial esophageal involvement (35). Thus, it is hypothesized that vonoprazan, at least partially, improved GERD symptoms in the patients with SSc compared to PPIs, due to the more efficient suppression of acid at night.

In the present study, only $50 \%$ of SSc patients on $10 \mathrm{mg}$ showed improvement. Generally, GERD treatment requires sufficient acid suppression, defined as an intra-gastric $\mathrm{pH}>4$ HTR within 83-91\% (33). However, with $10 \mathrm{mg} /$ day vonoprazan treatment, $\mathrm{pH}>4 \mathrm{HTR}$ was shown to be reached in $63.3 \%$ patients. In contrast, with $20 \mathrm{mg} /$ day vonoprazan treatment, $\mathrm{pH}>4 \mathrm{HTR}$ 
was reported to be reached in $83.4 \%$ of patients (33). Thus, in SSc patients with PPI-resistant GERD, top-down therapy, with a starting dose of $20 \mathrm{mg} /$ day vonoprazan, may be more appropriate than starting at $10 \mathrm{mg}$. Although the long-term effects of vonoprazan in SSc patients remains unknown, the symptoms of the patients on vonoprazan whom are being followed up (13 out of 15 patients) have been well controlled for $>2$ years.

Side effects of vonoprazan include nausea, vomiting, constipation, abdominal pain, skin rashes and heartburn, which are similar to those of classic PPIs (16). Although the long-term side effects of vonoprazan have not yet been fully elucidated, it has been reported to induce higher gastrin levels compared with PPIs, which has the potential to lead to enterochromaffin-like cell hyperplasia as well as potentiating the development of carcinoid tumors (36), hyperparathyroidism $(32,33,37)$ hypomagnesemia and hypocalcemia $(38)$. Considering these facts, careful monitoring of the development of endocrine cell tumors should be performed.

The present study found that the effects of vonoprazan were rather limited in the presence of dysphagia, which is a common symptom in patients with SSc (39). Although the reason for this tendency is unclear, it is hypothesized that vonoprazan may not sufficiently counteract the GERD symptoms in patients with a significant decrease in esophageal movement. Conversely, no association was found between the grade of SSc and the improvement rate of vonoprazan.

There are several limitations to the present study. The first is the design, as it was a single-arm, retrospective study with no comparison groups. The second limitation concerns the lack of patients with endoscopically severe esophagitis presented as LA grade C/D. Furthermore, there were variations in the observation period due to the retrospective nature of the study. A future large-scale prospective comparative study should be performed to confirm the results.

In conclusion, SSc patients with GERD, including those refractory to conventional PPI therapy, experienced significant relief from symptoms when treated with $20 \mathrm{mg}$ /day vonoprazan. The results demonstrate the efficacy of vonoprazan for the treatment of GERD in SSc patients with PPI-resistant symptoms, which may lead to an improvement in the patients quality of life.

\section{Acknowledgements}

This work was supported by Professor Atsushi Utani (deceased; Department of Dermatology, Nagasaki University Graduate School of Biomedical Science). We are deeply grateful to him for his advice and help with patient recruitment. We would also like to thank Dr Yuta Koike (Department of Dermatology, Nagasaki University Graduate School of Biomedical Science), Dr Naoki Iwamoto (Department of Immunology and Rheumatology, Nagasaki University Graduate School of Biomedical Science), Dr Tomoyuki Kakugawa (Department of Respiratory Medicine, Nagasaki University Graduate School of Biomedical Science) and Dr Masataka Umeda (Department of Immunology and Rheumatology, Nagasaki University Graduate School of Biomedical Science) for their clinical assistance.

\section{Funding}

No funding was received.

\section{Availability of data and materials}

The datasets used and/or analyzed during the present study are available from the corresponding author on reasonable request.

\section{Authors' contribution}

MT performed the collection and analysis of the data and wrote the manuscript. HM, YA, and KN designed the study, interpreted the data and finalized the manuscript. MA, TH, $\mathrm{KI}$, and AK collected the participants data and interpreted the data. MK, KH, KM, NY and FT interpreted the data. MT and HK performed the statistical analysis. All authors read and approved the final manuscript.

\section{Ethics approval and consent for participate}

The Nagasaki University Hospital Ethics Committee approved the present study protocol (approval nos. 16062704 and 17082135). All investigations were performed in accordance with the 1964 Declaration of Helsinki. The information was obtained only from anonymized electronic medical records. According to the guidelines of the Nagasaki University Hospital Ethical Committee's official disclosure system, if the subject of the study has minimal risk, the patient may choose to opt out of a study through our institution's homepage (mh.nagasaki-u. ac.jp/research/rinsho/patients/open.html). Informed consent was obtained from all patients in this study.

\section{Patients consent for publication}

All participants consented to the publication of relevant data following anonymization of personal information.

\section{Competing interests}

The authors declare that they have no competing interests.

\section{References}

1. Allanore Y, Simms R, Distler O, Trojanowska M, Pope J, Denton CP and Varga J: Systemic sclerosis. Nat Rev Dis Primers 1: 15002, 2015.

2. Ntoumazios SK, Voulgari PV, Potsis K, Koutis E, Tsifetaki N and Assimakopoulos DA: Esophageal involvement in scleroderma: Gastroesophageal reflux, the common problem. Semin Arthritis Rheum 36: 173-181, 2006.

3. Raja J, Ng CT, Sujau I, Chin KF and Sockalingam S: High-resolution oesophageal manometry and 24-hour impedance-pH study in systemic sclerosis patients: Association with clinical features, symptoms and severity. Clin Exp Rheumatol 34 (Suppl 100): S115-S121, 2016.

4. Luciano L, Granel B, Bernit E, Harle JR, Baumstarck K Grimaud JC, Bouvier M and Vitton V: Esophageal and anorectal involvement in systemic sclerosis: A systematic assessment with high resolution manometry. Clin Exp Rheumatol 34 (Suppl 100): S63-S69, 2016.

5. Vischio J, Saeed F, Karimeddini M, Mubashir A, Feinn R, Caldito G, Striegel K and Rothfield N: Progression of esophageal dysmotility in systemic sclerosis. J Rheumatol 39: 986-991, 2012.

6. Kaye SA, Siraj QH, Agnew J, Hilson A and Black CM: Detection of early asymptomatic esophageal dysfunction in systemic sclerosis using a new scintigraphic grading method. J Rheumatol 23: 297-301, 1996.

7. Roman S, Hot A, Fabien N, Cordier JF, Miossec P, Ninet J and Mion F; Réseau Sclérodermie des Hospices Civils de Lyon: Esophageal dysmotility associated with systemic sclerosis: A high-resolution manometry study. Dis Esophagus 24: 299-304, 2011. 
8. Sharma SK, Adarsh MB, Sinha SK, Bhattacharya A, Rana S, Singh S and Dhir S: The Gastrointestinal dysmotility and infections in Systemic Sclerosis-A real world scenario. Curr Rheumatol Rev 13: 1-1, 2017.

9. Lahcene M, Oumnia N, Matougui N, Boudjella M, Tebaibia A and Touchene B: Esophageal involvement in scleroderma: Clinical, endoscopic, and manometric features. ISRN Rheumatol 2011: 325826, 2011.

10. Arif T, Masood Q Singh J and Hassan I: Assessment of esophageal involvement in systemic sclerosis and morphea (localized scleroderma) by clinical, endoscopic, manometric and $\mathrm{pH}$ metric features: A prospective comparative hospital based study. BMC Gastroenterol 15: 24, 2015

11. Kimmel JN, Carlson DA, Hinchcliff M, Carns MA, Aren KA, Lee $\mathrm{J}$ and Pandolfino JE: The association between systemic sclerosis disease manifestations and esophageal high-resolution manometry parameters. Neurogastroenterol Motil 28: 1157-1165, 2016.

12. Liu X, Li M, Xu D, Hou Y, Wang Q, Tian Z, Sun Q and Zeng X: Prevalence and clinical importance of gastroesophageal reflux in Chinese patients with systemic sclerosis. Clin Exp Rheumatol 30 (2 Suppl 71): S60-S66, 2012.

13. Nagaraja V,McMahan ZH, Getzug T and Khanna D: Management of gastrointestinal involvement in scleroderma. Curr Treatm Opt Rheumatol 1: 82-105, 2015.

14. Muro Y, Sugiura K, Nitta Y, Mitsuma T, Hoshino K, Usuda T, Hayashi K, Murase Y, Shimizu M and Matsuo H: Scoring of reflux symptoms associated with scleroderma and the usefulness of rabeprazole. Clin Exp Rheumatol 27 (3 Suppl 54): S15-S21, 2009.

15. Marie I, Ducrotte P, Denis P, Hellot MF and Levesque H: Oesophageal mucosal involvement in patients with systemic sclerosis receiving proton pump inhibitor therapy. Aliment Pharmacol Ther 24: 1593-1601, 2006.

16. Akazawa Y, Fukuda D and Fukuda Y: Vonoprazan-based therapy for Helicobacter pylori eradication: Experience and clinical evidence. Therap Adv Gastroenterol 9: 845-852, 2016.

17. Hoshino S, Kawami N, Takenouchi N, Umezawa M, Hanada Y Hoshikawa Y, Kawagoe T, Sano H, Hoshihara Y, Nomura T and Iwakiri K: Efficacy of vonoprazan for proton pump inhibitor-resistant reflux esophagitis. Digestion 95: 156-161, 2017.

18. Sakurai Y, Mori Y, Okamoto H, Nishimura A, Komura E, Araki T and Shiramoto M: Acid-inhibitory effects of vonoprazan $20 \mathrm{mg}$ compared with esomeprazole $20 \mathrm{mg}$ or rabeprazole $10 \mathrm{mg}$ in healthy adult male subjects-a randomised open-label cross-over study. Aliment Pharmacol Ther 42: 719-730,2015.

19. Rickham PP: Human experimentation. Code of ethics of the world medical association. Declaration of Helsinki. Br Med J 2: 177, 1964.

20. Kusano M, Shimoyama Y, Sugimoto S, Kawamura O, Maeda M, Minashi K, Kuribayashi S, Higuchi T, Zai H, Ino K, et al: Development and evaluation of FSSG: frequency scale for the symptoms of GERD. J Gastroenterol 39: 888-891, 2004.

21. LeRoy EC, Black C, Fleischmajer R, Jablonska S, Krieg T, Medsger TA Jr, Rowell $\mathrm{N}$ and Wollheim F: Scleroderma (systemic sclerosis): Classification, subsets and pathogenesis. J Rheumatol 15: 202-205, 1988.

22. Danjo A, Yamaguchi K, Fujimoto K, Saitoh T, Inamori M, Ando T, Shimatani T, Adachi K, Kinjo F, Kuribayashi S, et al: Comparison of endoscopic findings with symptom assessment systems (FSSG and QUEST) for gastroesophageal reflux disease in Japanese centres. J Gastroenterol Hepatol 24: 633-638, 2009.

23. Johnson LF and Demeester TR: Twenty-four-hour pH monitoring of the distal esophagus. A quantitative measure of gastroesophageal reflux. Am J Gastroenterol 62: 325-332, 1974.

24. Kahrilas PJ, Bredenoord AJ, Fox M, Gyawali CP, Roman S, Smout AJ and Pandolfino JE; International High Resolution Manometry Working Group: The Chicago Classification of esophageal motility disorders, v3.0. Neurogastroenterol Motil 27: $160-174,2015$
25. Miwa H, Yokoyama T, Hori K, Sakagami T, Oshima T, Tomita T, Fujiwara Y, Saita H, Itou T, Ogawa H, et al: Interobserver agreement in endoscopic evaluation of reflux esophagitis using a modified Los Angeles classification incorporating grades $\mathrm{N}$ and M: A validation study in a cohort of Japanese endoscopists. Dis Esophagus 21: 355-363, 2008

26. Medsger TA Jr, Bombardieri S, Czirjak L, Scorza R, Della Rossa A and Bencivelli W: Assessment of disease severity and prognosis. Clin Exp Rheumatol 21 (4 Suppl 30): S42-S46, 2003.

27. Thonhofer R, Siegel C, Trummer M and Graninger W: Early endoscopy in systemic sclerosis without gastrointestinal symptoms. Rheumatol Int 32: 165-168, 2012.

28. Kusano M, Hosaka H, Kawada A, Kuribayashi S, Shimoyama Y, Kawamura O and Moki F: Development and evaluation of a modified frequency scale for the symptoms of gastroesophageal reflux disease to distinguish functional dyspepsia from non-erosive reflux disease. J Gastroenterol Hepatol 27: 1187-1191, 2012.

29. Kasugai K, Funaki Y, Izawa S, Ogasawara N and Sasaki M: Non-erosive reflux disease (NERD). Nihon Rinsho 74: 1351-1356, 2016 (In Japanese)

30. Miyazaki H, Igarashi A, Takeuchi T, Teng L, Uda A, Deguchi H, Higuchi $\mathrm{K}$ and Tango T: Vonoprazan versus proton-pump inhibitors for healing gastroesophageal reflux disease: A systematic review. J Gastroenterol Hepatol 34: 1316-1328, 2019.

31. Furuta T, Shirai N, Xiao F, Ohashi K and Ishizaki T: Effect of high-dose lansoprazole on intragastic $\mathrm{pH}$ in subjects who are homozygous extensive metabolizers of cytochrome P4502C19. Clin Pharmacol Ther 70: 484-492, 2001.

32. Kagami T, Sahara S, Ichikawa H, Uotani T, Yamade M, Sugimoto M, Hamaya Y, Iwaizumi M, Osawa S, Sugimoto K, et al: Potent acid inhibition by vonoprazan in comparison with esomeprazole, with reference to CYP2C19 genotype. Aliment Pharmacol Ther 43: 1048-1059, 2016.

33. Jenkins H, Sakurai Y, Nishimura A, Okamoto H, Hibberd M, Jenkins R, Yoneyama T, Ashida K, Ogama Y and Warrington S: Randomised clinical trial: Safety, tolerability, pharmacokinetics and pharmacodynamics of repeated doses of TAK-438 (vonoprazan), a novel potassium-competitive acid blocker, in healthy male subjects. Aliment Pharmacol Ther 41: 636-648, 2015.

34. Iwakiri K, Sakurai Y, Shiino M, Okamoto H, Kudou K, Nishimura A, Hiramatsu N, Umegaki E and Ashida K: A randomized, double-blind study to evaluate the acid-inhibitory effect of vonoprazan $(20 \mathrm{mg}$ and $40 \mathrm{mg}$ ) in patients with proton-pump inhibitor-resistant erosive esophagitis. Therap Adv Gastroenterol 10: 439-451, 2017.

35. Basilisco G, Carola F, Vanoli M, Bianchi P and Barbera R: Oesophageal acid clearance in patients with systemic sclerosis: Effect of body position. Eur J Gastroenterol Hepatol 8: 205-209, 1996.

36. Larsson H, Hakanson R, Mattsson H, Ryberg B, Sundler F and Carlsson E: Omeprazole: Its influence on gastric acid secretion, gastrin and ECL cells. Toxicol Pathol 16: 267-272, 1988.

37. Laria A, Zoli A, Gremese E and Ferraccioli GF: Proton pump inhibitors in rheumatic diseases: Clinical practice, drug interactions, bone fractures and risk of infections. Reumatismo 63: 5-10, 2011.

38. Perez MO, Neves EF, Bortolai CB, Sampaio-Barros PD, Andrade DC and Seguro LP: Hypomagnesaemia and hypocalcaemia in a patient with systemic sclerosis: Role of proton pump inhibitors. Clin Exp Rheumatol 32 (6 Suppl 86): S225-S227, 2014.

39. Sheehan NJ: Dysphagia and other manifestations of oesophageal involvement in the musculoskeletal diseases. Rheumatology (Oxford) 47: 746-752, 2008.

This work is licensed under a Creative Commons Attribution-NonCommercial-NoDerivatives 4.0 International (CC BY-NC-ND 4.0) License. 